\title{
THEORY OF OPTICALLY NONLINEAR EFFECTS IN ULTRAFAST SPATIO-TEMPORAL DYNAMICS OF SEMICONDUCTORS
}

\author{
A. KNorr, F. Steininger and S.W. Koch \\ Fachbereich Physik und Zentrum für MaterialwissenschaIten, Philipps-Universität \\ Renthof 5, 35032 Marburg, Germany
}

\begin{abstract}
UItrafast spatio-temporal effects in optically excited semiconductors are investigated by solving the coupled semiconductor Maxwell-Bloch equations which include the relevant relaxation phenomena. The analysis is used to describe transport of electronic excitations on nano- to micrometer scales with a dynamic range on the femto- to picosecond time scale.
\end{abstract}

PACS numbers: 78.47.+p, 78.66.-w

\section{Introduction}

Recently, the dynamics of excitons, biexcitons and the electron-hole plasma after excitation with femtosecond light pulses $\left(1 \mathrm{fs}=10^{-15} \mathrm{~s}\right)$ in semiconductor micro- and nanostructures has been studied extensively $[1,2]$. Coherent nonlinear effects such as the optical Stark-effect, four-wave mixing (FWM), photon echoes, and Rabi oscillations have been observed [3]. Whereas the linear optical susceptibility is dominated by electron-hole Coulomb (exciton) effects, at moderate intensities, biexcitons may form and carrier-carrier scattering takes place for the excitation of continuum states. For sufficiently high electron-hole densities in a semiconductor, negative absorption, i.e., optical gain appears [4].

The purpose of this paper is to give a short introduction in the theoretical treatment of spatio-temporal effects in semiconductor optics and to review some recent results. Our analysis includes light propagation effects in optically thick samples as well as optically induced electron and exciton transport.

\section{Observables and equations of motion}

Optically excited electron-hole-pair excitations decay via stimulated or spontaneous radiative recombination, thus emitting optical radiation which possibly interferes with the applied electromagnetic fields. The simplest example of a measurable quantity is the electromagnetic field intensity or intensity correlation functions. As usual, the electromagnetic field is represented by the scalar potential $\Phi$ 
and the vector potential $A$. In the transverse gauge $(\nabla \cdot A=0)$, the equation of motion for the vector potential is given by [5]

$$
\nabla^{2} A-\frac{1}{c^{2}} \frac{\partial^{2}}{\partial t^{2}} A=-\frac{4 \pi}{c_{0}} j_{\mathrm{T}}
$$

where $c$ is the velocity of light in the background medium and $j_{\mathrm{T}}$ is the transversal part of the current $j$

$$
j_{\mathrm{T}}(r)=\left[\frac{e}{m_{0}} \sum_{1,2} \varphi_{1}^{*}(r)\left(p-\frac{e}{c_{0}} A\right) \varphi_{2}(r) a_{1}^{\dagger} a_{2}\right]_{\mathrm{T}} .
$$

Here, the material operators are the Fermionic annihilation and creation operators $a_{1}, a_{1}^{\dagger}$, which annihilate and create electrons in the eigenstates $\varphi_{1}$ of the single particle material Hamiltonian. This describes the motion of a single valence electron in a periodic lattice potential plus the additional confinement potential of the quantum structure. Thus the electron index 1 is a compound quantum number which includes subband, band and wave number $1=m_{1}, \lambda_{1}, k_{1}$.

The equations of motion for the expectation value of the current matrix $\left\langle a_{1}^{\dagger} a_{2}\right\rangle$ have to be derived from the full Hamiltonian [5, 3], which includes the scalar potential $\Phi$ via the longitudinal Coulomb coupling between the carrier states $\varphi_{1}$ as well as the coupling to the vector potential $\boldsymbol{A}$. To derive the equations of motion for the quantum mechanical expectation values of $\left\langle a_{1}^{\dagger} a_{2}\right\rangle=\operatorname{tr}\left(a_{1}^{\dagger} a_{2} \sigma\right)$, we apply a projection formalism [6] to the von Neumann equation for the density operator $\sigma$

$$
\frac{\mathrm{d}}{\mathrm{d} t} \sigma=-\mathrm{i}[H, \sigma]=-\mathrm{i} \widehat{L} \sigma \text {. }
$$

As a result we obtain equations of motion for the relevant density matrix $\sigma_{12}=$ $\operatorname{tr}\left(a_{1}^{\dagger} a_{2} \sigma_{\text {rel }}\right)=\operatorname{tr}\left(a_{1}^{\dagger} a_{2} \sigma\right)$. For the purpose of coherent optics it is convenient to use the canonical non-equilibrium and non-stationary density operator $\sigma_{\text {rel }}$ as

$$
\sigma_{\text {rel }}=\frac{\exp \left(-\sum_{1,2} \lambda_{12}(t) a_{1}^{\dagger} a_{2}\right)}{\operatorname{tr}\left(\exp \left(-\sum_{1,2} \lambda_{12}(t) a_{1}^{\dagger} a_{2}\right)\right)}
$$

where we have chosen for the observation space the observables which determine the current via Eq. (2). We need here to include all possible combinations of two-particle operators $a_{1}^{\dagger} a_{2}$. The quantities $\lambda$ in Eq. (4) are the usual Lagrange-multipliers which contain the full time dependence of the system [6]. The projection operator formalism divides the equation for the relevant density matrix into a mean-field and a correlation part. The mean-field part contains the usual Hartree-Fock contributions whereas the correlation part describes, depending on the approximations made, higher bound Coulomb states such as biexcitons or Coulomb scattering. In this paper we use a two-band model $(\lambda=v, c)$ excited with a polarized single beam. Under these conditions it is sufficient to consider Coulomb scattering contributions only and to neglect higher bound states occurring in two polarization calculations [7].

\section{Light pulse propagation}

In this section, we investigate the propagation of plane wave femtosecond pulses through extended bulk semiconductors. Due to the interaction with the 
material, the pulse $\boldsymbol{E}(r, t)=-\frac{1}{c_{0}} \dot{\boldsymbol{A}}$ accumulates temporal deformations which contain information on the light-material interaction. For plane wave propagation, the equation for the forward propagated electric field envelope reads

$$
\frac{\partial E}{\partial \xi}=-\frac{2 \pi}{n c_{0}} j(\eta)
$$

where $j=-\mathrm{i} \omega_{\mathrm{gap}}\left(d_{c v} / V\right) \sum_{1,2} \sigma_{k_{1}, k_{2}}^{v c}$. The iteration scheme for the field is equivalent to a slowly varying envelope equation in semiconductors [8]. To describe the propagation self-consistently, we have to derive the material equations for $\sigma_{12}$. Non-propagating electronic excitations are characterized by a vanishing momentum $Q=k_{1}-k_{2}$, i.e., $\sigma_{k_{1} k_{2}}^{v c}=\sigma_{k_{1}}^{v c} \delta_{k_{1}, k_{2}}$. We use the electron-hole notation where the interband current dynamics is introduced as $P_{k}=\sigma_{k k}^{v c}=\left\langle a_{v, k}^{\dagger} a_{c, k}\right\rangle$. This quantity couples to the occupation numbers for electrons and holes, $f_{k}^{e}=\left\langle a_{c, k}^{\dagger} a_{c, k}\right\rangle$, $f_{k}^{\mathrm{h}}=1-\left\langle a_{v, k}^{\dagger} a_{v, k}\right\rangle$. The mean-field contributions (MFCs) read

$$
\begin{aligned}
& -\left.\mathrm{i} \dot{P}_{k}\right|_{\mathrm{MF}}=\left(\varepsilon_{k}^{\mathrm{h}}-\varepsilon_{k}^{\mathrm{e}}\right) P_{k}+\Omega_{k}\left(1-f_{k}^{\mathrm{e}}{ }_{k}-f^{\mathrm{h}}{ }_{k}\right), \\
& -\left.\mathrm{i} \dot{f}_{k}^{\mathrm{e} / \mathrm{h}}\right|_{\mathrm{MF}}=\left(\Omega_{k} P_{k}^{*}-\Omega_{k}^{*} P_{k}\right),
\end{aligned}
$$

where we have defined a generalized Rabi-frequency $\Omega_{k}$ and renormalized energy transitions $\varepsilon_{k}$. It can be recognized that the MFCs show significant differences in comparison with the two level Bloch equations [9]. The optical transitions are inhomogeneously broadened due to the different electronic wave number states $k$. Moreover, the Rabi-frequency and the energies are renormalized by many particle Coulomb contributions and can be written as a renormalization of single particle properties. They yield a decrease in the effective single particle energy for populated electron-hole states and an amplification of the external field by the Coulomb induced field of all $k$ dependent oscillators [3]. The correlation contributions (CCs) read

$$
\begin{aligned}
& \left.\frac{\partial P_{k}}{\partial t}\right|_{\text {corr }}=-\Sigma_{\mathrm{d}}(k) P_{k}+\sum_{q} \Sigma_{\mathrm{nd}}(k, q) P_{k+q} \ldots, \\
& \left.\frac{\mathrm{d} f_{k}^{\mathrm{e} / \mathrm{h}}}{\mathrm{d} t}\right|_{\text {corr }}=\Gamma_{\text {in }}^{\mathrm{e} / \mathrm{h}}(k)\left(1-f_{k}^{\mathrm{e} / \mathrm{h}}\right)-\Gamma_{\text {out }}^{\mathrm{e} / \mathrm{h}}(k) f_{k}^{\mathrm{e} / \mathrm{h}} \cdots,
\end{aligned}
$$

where $\Sigma$ denotes diagonal and nondiagonal polarization dephasing and $\Gamma$ is the expression for in- and out-scattering in the equation for the distribution functions [10]. At a level beyond mean-field theory, these contributions cannot be written as energy or field renomalizations. The CC leads to the interference of different single particle oscillations that result in optical dephasing of the total polarization and carrier relaxation.

The material equations describe the field induced transitions $P_{k}$ and the population probability $f_{k}^{\mathrm{e} / \mathrm{h}}$ of the states in a semiconductor medium. Because of the Coulomb-interaction contained in the MFC the formation of bound states (excitons) and scattering states (free electron-hole pairs) takes place (linear optics). Each exciton corresponds to a strong peak in the optical susceptibility below the band gap whereas the continuum is more or less structureless [3]. In the nonlinear 
optical response, finite electron-hole densities $f_{k}^{e / h}$ can be generated and the interaction with the optical field is influenced via the Pauli-blocking factor $1-f^{\mathrm{e}_{k}}-f^{\mathrm{h}}{ }_{k}$ and the Coulomb renormalizations. If the blocking factor is negative, the system switches from the absorbing to the gain state, where light amplification is possible.

\subsection{Polaritons and polariton-scaltering}

We first investigate the interaction of a weak optical pulse with the xcitonic resonance in GaAs. For this purpose, the initial pulse width FWHM $=400 \mathrm{fs}$ (full width at half intensity maximum) is chosen to excite only the first $(1 s)$ exciton. Figure la shows on a logarithmic scale the temporal pulse intensity $I / I_{0}$ for different propagation distances. At a distance of $z=0.2 \mu \mathrm{m}$, i.e., shortly after the entrance in the sample, one recognizes that the leading edge of the pulse is absorbed and reemitted after the trailing edge. For a larger propagation length $(10 \mu \mathrm{m})$, the transmitted pulse develops strong temporal oscillations.
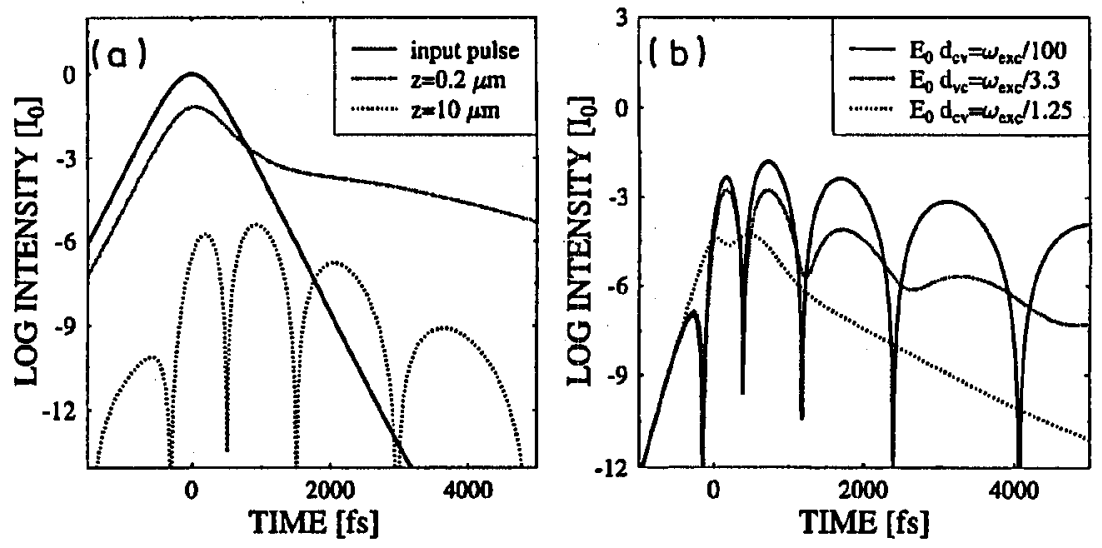

Fig. 1. (a) Temporal pulse intensity of an initially $400 \mathrm{fs}$ (FWHM) $(z=0)$ light pulse after propagation over $z=0.2,10 \mu \mathrm{m}$ of GaAs bulk material. (b) Temporal pulse intensity of an initially $400 \mathrm{fs}$ (FWHM) $(z=0)$ light pulse after propagation over $z=2 \mu \mathrm{m}$ of GaAs bulk material for different input fields $E_{0}$. The polariton beating is washed out due to the excitation-induced dephasing.

This shape modulation is caused by the frequency dependent group velocity modifications due to the strong refractive index in the vicinity of the excitonic resonance. Therefore, different spectral polariton components arrive at different times at the end of the sample and interfere with each other resulting in a pronounced temporal interference pattern. To investigate the intensity dependence of the polariton beating we have varied the strength of the input pulse intensity. Figure $1 \mathrm{~b}$ shows the intensity dependence at $z=2 \mu \mathrm{m}$. It can be recognized that for increasing input intensity the signal decreases. The polariton beating gradually washes out and vanishes completely for strong fields. The decay of the coherent signal results from the excitation induced dephasing and energy fluctuations of 
the excitonic coherence via the scattering with excitons and electron-hole pairs. Such polariton beating effects and their excitation dependence have been recently observed experimentally, for a detailed study compare [11].

\subsection{High intensity off-resonant propagation}

After the discussion of resonant pulse propagation we investigate high intensity propagation of a short optical pulse (FWHM=100 fs) well below the band edge. The situation for thin samples has widely been studied in the investigation of the optical Stark-effect, where the spectral response of the excitonic resonance to an off-resonant light field is investigated [12]. In general, off-resonant excitation yields to an adiabatic response of the semiconductor if the detuning $\Delta$ of carrier frequency and optical transitions is much larger than the inverse pulse width $\Delta \gg \mathrm{FWHM}^{-1}$. In this case, the electron-hole density follows the shape of the pulse envelope [9]. In the following, we investigate the interaction of a semiconductor with a high intensity pulse (FWHM $=100 \mathrm{fs}$, pulse area $\int \mathrm{d} t d_{c v} E(t)=6 \pi$ ) with a carrier frequency corresponding to 10-Rydberg energies below the excitonic resonance where adiabatic following of the electron density is expected. Figure 2a shows that the electron density exhibits a maximum at $t=0$, where the light pulse peaks and decreases but remains finite after the pulse. The adiabatic following of the electron density with respect to the pulse envelope is not complete because of the finite background and carrier induced dephasing. With increasing propagation length, we observe strong temporal density oscillations which lead to a temporal break-up of the pulse intensity, compare Fig. $2 \mathrm{~b}$. It can be recognized that the
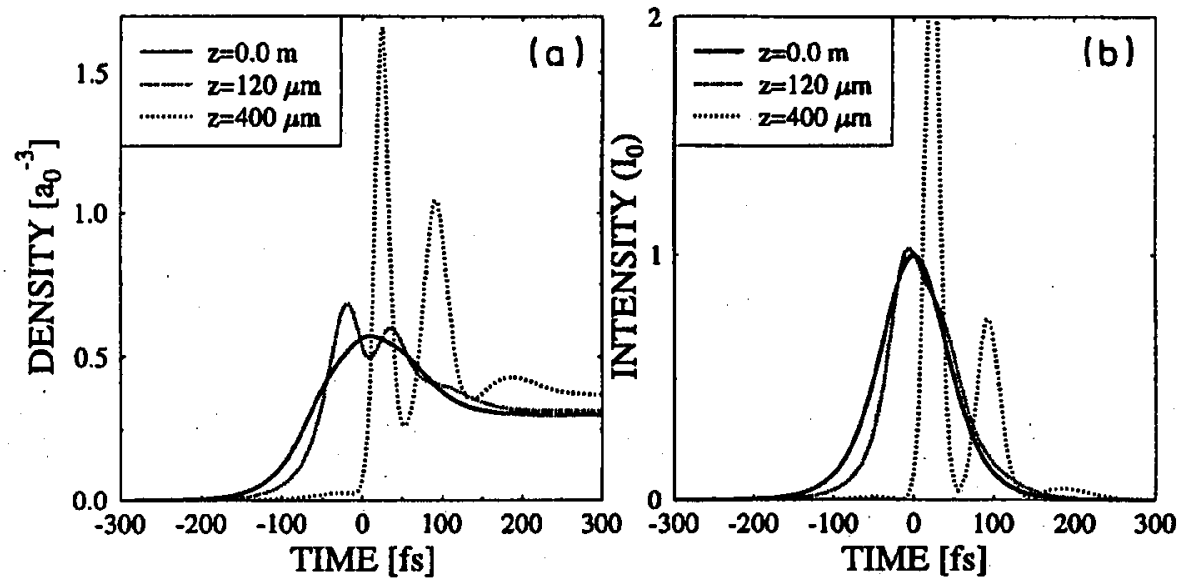

Fig. 2. (a) Electron-hole density as a function of time for excitation with an initially $100 \mathrm{fs}$ (FWHM) pulse at different distances in the sample. (b) Pulse intensity. Chirp induced pulse break-up is observed.

pulse break-up is initiated by the oscillations in the electron density. Comparing density and intensity plots at $z=120 \mu \mathrm{m}$ it can be recognized that the density shows oscillations whereas the pulse is still unmodulated. The electron density 
oscillations correspond to a series of absorption (increasing density) and amplification (decreasing density) which manifest itself in modulations of in the pulse shape for larger propagation distance. The temporal modulation of the density itself is propagation induced. The analysis of the numerical data shows that the density oscillations occur at a propagation distance where the limit of adiabatic following is violated by the propagation induced phase change $\dot{\phi}(t)$ of the optical pulse. $\dot{\phi}$ is the so-called frequency chirp which determines the instantaneous pulse frequency $\omega_{l}+\dot{\phi}$ at time $t$. At the spatial distance of approximately $100 \mu \mathrm{m}$ where the density modulation occurs, the induced frequency shift compensates the initial detuning of 10-Rydberg energies of the pulse and drives a fast density dynamics which cannot be overcompensated by the initial detuning of the pulse. This effect has been experimentally observed in semiconductor wave guide structures at room temperature [13].

\section{Propagation of material excitations}

\subsection{Transport of electron-hole pairs: phonon limited propagation}

In this section we study the propagation of optically generated electron wave packets in a quantum well under the influence of collisions with a phonon reservoir. Our calculation includes the propagation on femtosecond time scales where ballistic motion of electrons occurs [14] up to several picoseconds. After that time electron-phonon scattering dominates the propagation dynamics and a diffusive type of motion sets in. The field equation is solved in the paraxial limit

$$
E_{\mathrm{T}}=E_{\mathrm{T}, 0}-\frac{2 \pi}{n c_{0}} j
$$

where $E_{\mathrm{T}, 0}$ is the incident spatially localized excitation. Applying a bath approximation to the phonon system, the CCs for the polarization functions read

$$
\begin{aligned}
& \left.\frac{\partial}{\partial t} P_{k_{1}, k_{2}}\right|_{\text {phon }}=\sum_{q}\left[\left(w_{k_{1} \rightarrow k_{1}+q}^{v}+w_{k_{2}+q \rightarrow k_{2}}^{c}\right) P_{k_{1}+q, k_{2}+q}\right. \\
& \left.-\left(w_{k_{1}+q \rightarrow k_{1}}^{v}+w_{k_{2} \rightarrow k_{2}+q}^{c}\right) P_{k_{1}, k_{2}}\right]
\end{aligned}
$$

where $w$ are the nonlocal phonon scattering rates. Similar equations can be derived for the distribution functions [15]. We numerically solve these equations for a situation, where a focused laser pulse $(1 \mu \mathrm{m})$ generates a localized polarization and electron distribution. Since we are interested in the situation, where the electron-phonon and not the electron-photon interaction determines the dynamics, the pulse length is chosen to be short in comparison to the characteristic electron-phonon scattering time.

Figures 3a, b show the computed spatial profiles of the electronic density as a function of the radial distance $\left|r_{\|}\right|$from the beam focus for different times after the optical pulse and for two different temperatures $(T=0,300 \mathrm{~K})$. The insets display the energy distribution of the electronic excitation for times, where a quasi stationary energetical distribution has been reached after a relaxation process of the optically generated non-equilibrium excitation. We see that for both temperatures the initially generated non-equilibrium energy distribution develops 

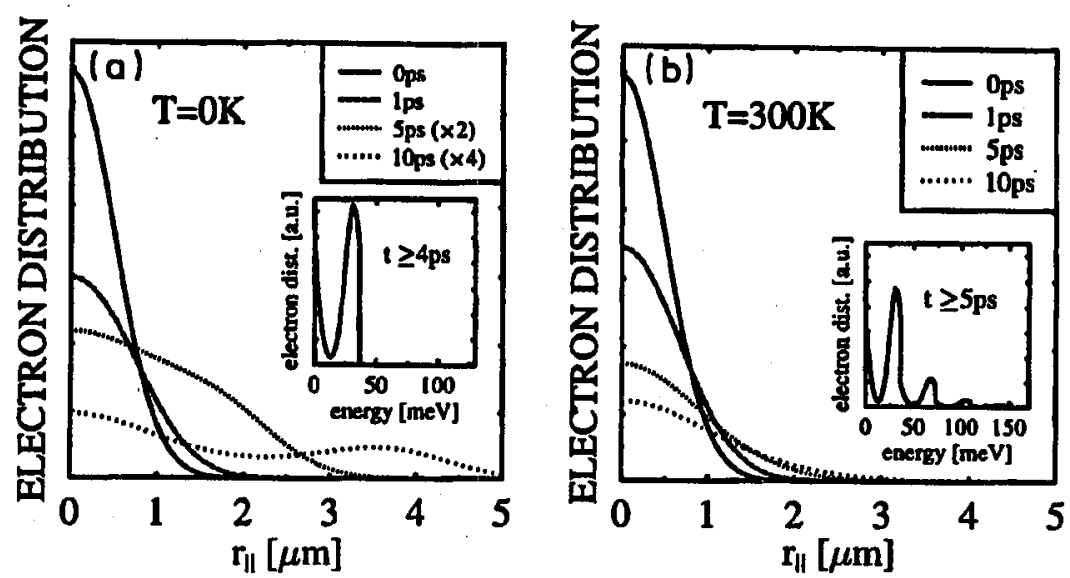

Fig. 3. (a) Spatial distribution of electron density for different times $t$ after the pulse at zero temperature and for excitation above the phonon threshold. The inset shows the stationary energy distribution of the electrons. (b) The same as in (a) but at a temperature $T=300 \mathrm{~K}$.

a number of energetically separated phonon replica. Note that although the energetical distribution does not change in time anymore, electron-phonon scattering takes place and affects the spatial motion of the electrons. In a free particle picture, i.e., without the electron-phonon interaction, the energy distribution is a measure of the absolute value of the electron velocity. For $T=0 \mathrm{~K}$ (Fig. 3a) two distinct phonon replica are observed in the stationary energy distribution. The spatial peak located at the center of the laser focus corresponds to the low energy peak of the energy distribution. The second spatial peak which develops after approximately $8 \mathrm{ps}$, moves consistently with the velocity distribution of the high energy peak of the electronic distribution.

The situation is well described within the picture of ballistic electron propagation. As a consequence of the enhanced electron-phonon interaction at higher temperatures $(T=300 \mathrm{~K})$ several phonon replica appear in the quasi-stationary energy distribution (see inset in Fig. 3b). Consequently, the electronic velocity distribution of a free particle wave packet involves several peaks separated by the phonon energy. On the other hand, for higher temperatures, the corresponding wave packet peaks for the different center velocities at the phonon replica do not develop. The spatial distribution exhibits only weak broadening with increasing time. These features cannot be understood solely from the energetic distribution of the electrons, since that distribution depends only on the absolute value of the velocity. A numerical analysis shows that for higher temperature the distribution of the angle between the single particle velocity and the position vector directed out of the focus is smeared out by the electron-phonon interaction. The direction of the electron motion is more and more randomized with increasing time and thus counteracting the ballistic motion [15]. 
The simplest analysis of the situation can be given for optical excitation having a carrier frequency directly at the semiconductor band edge. Here, the electronic wave packet is localized around the focus and is less structured in real space for lower temperatures than for above band edge excitation. We analyze the results within the mean square displacement (MSD) of the distribution $\Delta^{2}(t)=$ $N^{-1} \int \mathrm{d} r_{\|}^{2} r_{\|}^{2} f^{e}\left(r_{\|}\right)$and $\Delta_{0}=\Delta(t=0)$. The MSD can be used to characterize a motion as ballistic $\Delta^{2}-\Delta_{0}^{2} \propto t^{2}$, diffusive $\Delta^{2}-\Delta_{0}^{2} \propto t$. Figure 4 shows a temperature dependent cross-over from a regime with $\Delta^{2}-\Delta_{0}^{2} \propto t^{2}$ at earlier times to a regime with $\Delta^{2}-\Delta_{0}^{2} \propto t$ at larger times, thus indicating the transition of ballistic to diffusive wave packet propagation for band edge excitation. The temperature dependence of the cross-over time $t_{\mathrm{c}}$ between the two regimes is shown in the inset of Fig. 3 as a function of temperature. Note that the temperature dependence of the cross-over time can be fitted quite well with the inverse of the phonon distribution function $t_{\mathrm{c}} \propto n_{0}(T)^{-1}$ as can be seen in the inset.

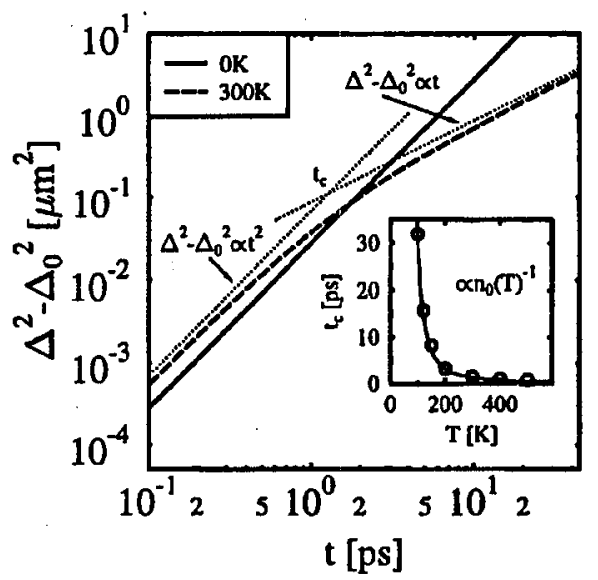

Fig. 4. Temporal behavior of the MSD of the excited electron density for different temperatures $(T=0 \mathrm{~K}$ and $T=300 \mathrm{~K}$ ). The time resolved cross-over from ballistic to diffusive behavior is observed. The inset shows the cross-over time $t_{c}$ as a function of the temperature proportional to $n_{0}(T)^{-1}$.

\subsection{Surface polariton-transport: near-field optics}

In this section, we focus on the near-field response of excitons in a quantum well (QW). The idea of near-field optical microscopy is to increase the spatial resolution of conventional microscopy by using an object smaller than the wavelength of light as source or detector of electromagnetic radiation [16]. Most of todays near-field microscopes consist of a fiber tip, smaller than the wavelength of light, which can act as near-field emitter or collector if the sample is close enough to the tip. In a quantum well, where the motion of the electronic excitation is restricted to the plane $(z=0)$ of a thin layer we approximate the current as

$$
j_{\mathrm{QW}}=-\mathrm{i} \omega_{0} d_{v c}^{\mathrm{QW}} \sigma_{v c}^{\mathrm{QW}}(Q, \omega) \frac{\delta(z)}{A}
$$


where the density matrix is reduced to the $1 s$ exciton state. To not overload the analysis, we study a model system, where a point-like near-field source excites only longitudinal excitons. Here, the propagation vector and the polarization of the exciton are parallel to each other in the plane of the well. For heavy holes the dipole moment of the excitons has no $z$-component and the field equation Eq. (1) can be solved in Fourier-space

$$
\begin{gathered}
E_{\mathrm{T}}^{+}(Q, z, \omega)=-\frac{2 \pi}{c_{0}} j_{Q \mathrm{~W}}^{+}\left(\mathrm{e}^{\mathrm{i} \kappa z} \frac{\kappa}{k_{l}}-\mathrm{ie}^{-Q z} \frac{Q}{k_{l}}\right) \\
-\frac{2 \pi}{c_{0}} j_{\mathrm{NF}}^{+}\left(\mathrm{e}^{-\mathrm{i} \kappa\left(z-z_{0}\right)} \frac{\kappa}{k_{l}}-\mathrm{ie}^{Q\left(z-z_{0}\right)} \frac{Q}{k_{l}}\right)
\end{gathered}
$$

where $\kappa^{2}=\omega^{2} / c^{2}-Q^{2}$ and $k_{l}$ denotes $\omega / c \approx \omega_{l} / c$ within the slowly varying envelope appoximation (SVEA) and $Q=|Q|$ is the Fourier variable in plane of the well. Using the self-consistent transversal field the dispersion relation for the exciton $\sigma_{v c}^{\mathrm{QW}}(Q, \omega)$ coupled to the radiation field only is obtained

$$
D_{\mathrm{QW}}(Q)=\omega_{\mathrm{QW}}(Q)+\Gamma_{\mathrm{W}}\left(\sqrt{\left(Q / k_{l}\right)^{2}-1}-Q / k_{l}\right)
$$

Here, $\Gamma_{W}$ denotes the coupling constant of the well and the light field. The dispersion reproduces for $Q / k_{l} \rightarrow \infty$ the limit of free propagating excitons. For excitons having smaller wave vectors than $\omega_{0} / c\left(Q / k_{l}<1\right)$ the square-root in Eq. (14) becomes imaginary, thus describing excitons which decay radiatively into modes of the electromagnetic field. The excitons with $Q / k_{l}>1$ are called non-radiative because they do not decay into photons [17]. Figure 5 shows the spatial distribution of the excitonic density at different times after the switch on of the near-field source. It can be recognized that at $t>0$ a peaked exciton distribution at the center of the excitation is found. At larger times, the distribution expands out of the excitation region. It can be recognized that the envelope of the packet develops a spatially oscillating structure. The oscillation frequency decreases for increasing times at a fixed position and increases with propagation distance for a fixed time. The reason for the built-up of a wave packet and its characteristic propagation dynamics can be traced back to the excitation of excitons with different wave numbers $Q$ by the near-field source. In a simplified picture, the excitons propagate after their excitation according to their dispersion relation Eq. (14). Hence, excitons with $Q<k_{l}$ decay radiatively whereas non-radiative states $Q>k_{l}$ may propagate long distances without radiative decay. Thus, all excitons propagating on a longer time scale than $\Gamma_{\mathrm{W}}^{-1}$ have at least a velocity determined by the momentum $Q=k_{l}$. This limit determines the front of the trailing edge of the wave packet. The leading edge of the wave packet is determined by the fastest excitons excited by the near-field source. A numerical analysis shows that the spatial oscillations on the leading edge of the wave packet can be understood by superposition of exciton at $Q \approx k_{l}$ and free exciton at $Q \gg k_{l}$. 


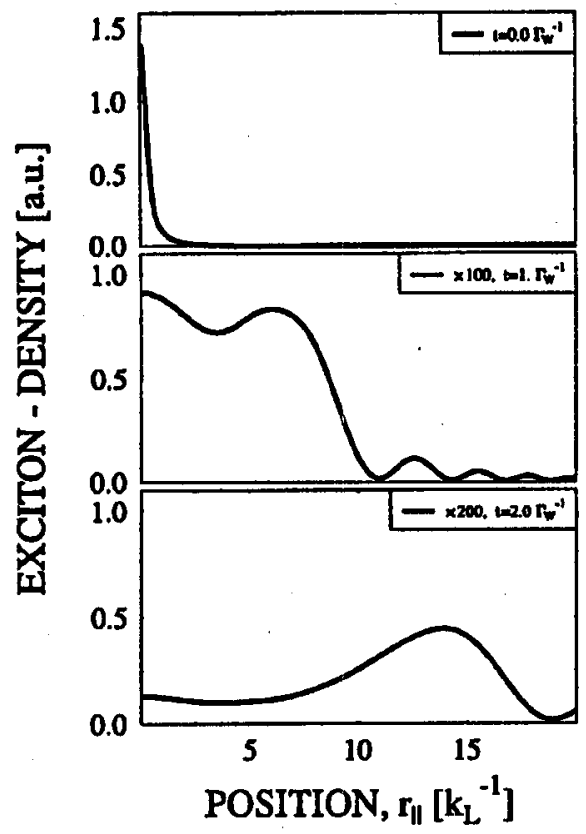

Fig. 5. Spatial distribution of the excitonic density at different times after an excitation with a point like near-field source. Time and space are normalized in units of the wave vector of light at the excitonic transition $\approx \frac{2 \pi}{300 \mathrm{~nm}}$ and the lifetime of an radiative exciton in a quantum well $(\approx 10 \mathrm{ps})$.

\section{Summary}

In conclusion, we have presented self-consistent solutions of the MaxwellBloch equations for spatio temporal dynamics in semiconductors. Our analysis includes light as well as electronic propagation phenomena and allows us to describe the transition from linear polariton propagation to nonlinear polariton scattering for far- and near-field optics.

\section{Acknowledgments}

We thank P. Thomas and B. Hanewinkel for useful discussions and the DFG (Germany) for financial support in the Semiconductor Quantum Coherence Program.

\section{References}

[1] See e.g. Procs. of the Conf. Optics of Excitons in Confined Systems in J. Phys. IV (Paris) 3 (1993) and Nuovo Cimento D 17, (1995).

[2] Procs. of the Confs. Nonlinear Optics and Excitation Kinetics in Semiconductors in Phys. Status Solidi B 159, No. 1 (1989); Phys. Status Solidi B 173, No. 1 (1992); Phys. Status Solidi B 188, No. 1 (1994).

[3] H. Haug, S.W. Koch, Quantum Theory of the Optical and Electronic Properties of Semiconductors, 3rd ed., World Scientific, Singapore 1994. 
[4] W.W. Chow, S.W. Koch, M. Sargent III, Semiconductor-Laser Physics, Springer, Berlin 1994.

[5] G. Mahan, Many Particle Physics, Plenum Press, New York 1990.

[6] E. Fick, G. Sauermann, The Quantum Statistics of Dynamical Processes, in Springer Series in Solid State Physics, Springer, Berlin 1990.

[7] W. Schäfer, D.S. Kim, J. Shah, T.C. Damen, J.E. Cunningham, K.W. Goossen, L.N. Pfeiffer, K. Köhler, Phys. Rev. B 53, 16429 (1996).

[8] W. Schäfer, K. Henneberger, Phys. Status Solidi B 159, 59 (1990); A. Knorr, R. Binder, M. Lindberg, S.W. Koch, Phys. Rev. A 46, 7179 (1992).

[9] L. Allan, J.H. Eberly, Optical Resonance in Two Level Systems, John Wiley and Sons, New York 1975.

[10] M. Lindberg, S.W. Koch, Phys. Rev. B 38, 3342 (1988); W. Schäfer, I. Brener, W. Knox, in: Coherent Optical Interactions in Semiconductors, Ed. R.T. Philipps, Plenum Press, New York 1994; A. Knorr, T. Stroucken, S. Hughes, S.W. Koch, Chem. Phys. 210, 27 (1996).

[11] D. Fröhlich, A. Kulik, B. Uebbing, A. Myssyrowicz, V. Langer, H. Stolz, W. von der Osten, Phys. Rev. Lett. 67, 2343 (1991).

[12] C. Ell, J.F. Müller, K. El Sayed, H. Haug, Phys. Rev. Lett. 62, 306 (1989).

[13] P.A. Harten, A. Knorr, J.P. Sokoloff, F. Brown de Colstoun, S.G. Lee, R. Jin, E.M. Wright, G. Khitrova, H.M. Gibbs, S.W. Koch, N. Peyghambarian, Phys. Rev. Lett. 69, 852 (1992).

[14] P. Kral, J. Masek, Acta Phys. Pol. A 82, 697 (1992); F. Steininger, A. Knorr, T. Stroucken, P. Thomas, S.W. Koch, Phys. Rev. Lett. 77, 550 (1996).

[15] F. Steininger, A. Knorr, P. Thomas, S.W. Koch, Z. Phys. B 103, 45 (1997).

[16] Near-Field Optics, Eds. D.W. Poll, D. Courjon, NATO ASI Series E: Applied Sciences, Vol. 242, Kluwer Academic Publ., Dordrecht 1991.

[17] C.L. Andreani, in: Confined Electrons and Photons: New Physics and Devices, Eds. E. Burstein, C. Weisbuch, Plenum Press, New York 1994. 